\title{
The spectrum of the fetal phonocardiogram as an indicator of fetal maturity
}

\author{
Joachim H Nagel
}

\section{Introduction}

Relatively few techniques offer a non-invasive means of investigating the fetus. Thus the expansion of diagnostic opportunities is a great challenge for biomedical engineering. Instead of searching for new, complicated measuring techniques, we have been trying to meet this challenge by developing suitable procedures aimed at improving the analysis of the data provided by present routine examinations. One highly promising result of our work has been the discovery that the power spectrum of the fetal phonocardiogram (PCG) can be used as a reliable indicator of fetal maturity.

\section{Physical basis of the PCG}

In phonocardiography the mechanical energy of the heart sounds is picked up by a microphone. At present the only reason for analysing the PCG is to evaluate the heart rate and determine various electromechanical time intervals. In this way the function of the fetal cardiovascular system may be monitored. If we consider the generation of the heart sounds from a more physical standpoint, it seems obvious that more information about the heart must be contained in the PCG than merely the time course of the contractions. Because the heart is a rather complex oscillation system, the PCG is a compound signal whose spectral power distribution is determined by numerous factors such as the various valves, the heart muscle and the intracardiac blood volume. Even without a knowledge of all the interactions involved, we may suppose that variations in any one of these parameters will lead to a shift of the PCG spectrum.

In order to obtain an idea as to the effects that may result, we may consider the vibration of the heart valves. In a very simple model the valves may be compared to a stressed membrane, the two-dimensional equivalent of a vibrating string. The resonance frequencies of a square membrane are given by the equation:

$$
\nu_{h, k}=\frac{1}{21} \sqrt{(1+h)^{2}+(1+k)^{2}} \cdot \sqrt{\frac{S}{\rho}}
$$


where $1=$ edge length, $\rho=$ density, $S=$ tension $h, k=$ series of the integer numbers $0,1,2,3 \ldots$

The fundamental frequency is:

$$
\nu_{\infty}=\frac{\sqrt{2}}{21} \cdot \sqrt{\frac{\mathrm{s}}{\rho}}
$$

Figure 1 shows the fundamental frequency in relation to the edge length for different tensions. Variations in the frequency caused by changing parameters can be

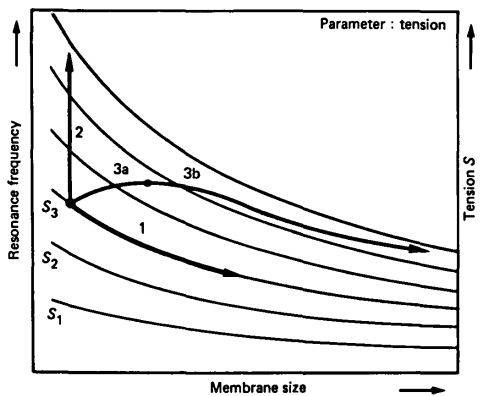

Figure 1 . Fundamental frequency of a stressed membrane dependent on size and tension

investigated. For a given tension, an increase in the dimensions of the membrane results in a decrease in frequency (trace 1). Increasing tension at a constant membrane size augments the resonance frequency (trace 2). Changes in both parameters complicate the course of the frequency curve. When the influence of increasing tension predominates, the frequency increases (3a), whereas predominance of an increase in the membrane dimensions results in a decrease in the vibration frequency (3b). In each individual case there is an unequivocal relationship between frequency, tension and membrane size.

\section{Spectral changes of the PCG with fetal maturity}

Applying these findings to the fetal heart valves, and assuming that during pregnancy both heart valves are growing and the contractile strength of the cardiac muscle is increasing (i.e. membrane tension is being augmented), we would expect to find spectral changes in the PCG as a function of the stage of fetal maturity. It is, of course, not realistic to describe the fetal heart in such a simple way - there are too 


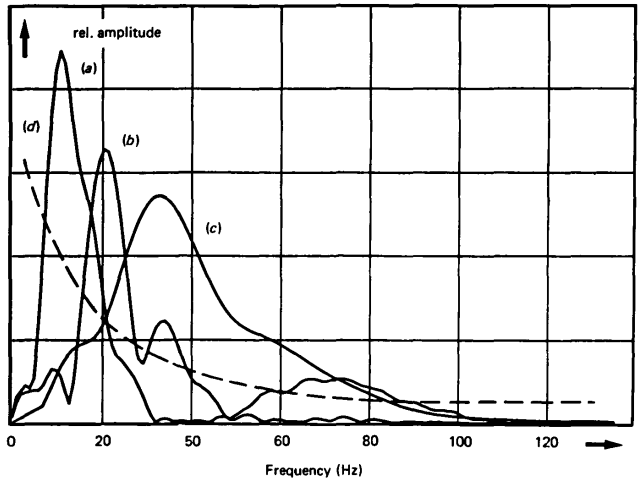

Figure 2. Frequency spectrum of the abdominal PCG. (a) maternal signal: (b) first heart sound of the fetus; (c) second heart sound of the fetus; (d) background noise

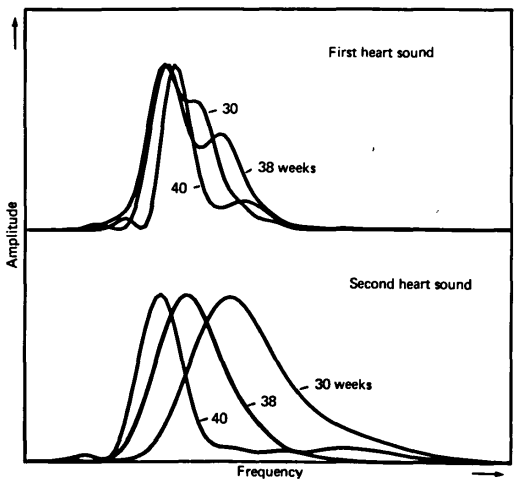

Figure 3. PCG spectra from different gestational ages 
many differences between this simplified model and the complex structure and function of the heart. Nevertheless, the tendency for spectrum changes to occur as a function of fetal growth should be apparent. In order to verify this assumption and to investigate the course of the PCG spectrum during pregnancy, extensive experimental studies have been necessary. Our initial findings showed that the conventional phonotransducers did not have suitable transmission characteristics. In order to pick up the PCG over its full bandwidth we first had to develop an improved microphone.

Spectral analysis was effected by discrete Fourier transformation of the abdominal signals which were recorded unfiltered. Prior to the transformation the PCG was broken down to obtain the separate spectra of the maternal background sounds, the first and second fetal heart sounds, and noise. Figure 2 shows a typical example of such a power spectrum. From the membrane model a clear shift of the fetal spectra would be expected at different stages of maturity. Differences can indeed be seen (Figure 3). Unfortunately, however, the location of the maxima does not always reveal an unequivocal systematic shift.

A reconsideration of the model makes clear the reason for this discrepancy. The previous assumption is valid only for one characteristic frequency of one single valve, whereas the PCG results from the superimposition of many components of the heart's complex oscillation system, each with a different vibration frequency and amplitude. The maximum power density is determined by the distribution of the characteristic frequencies and need not necessarily express the changes in the individual vibration modes. But, in every case, an increasing frequency of the individual oscillators shifts the mean value of the PCG spectrum towards higher frequencies. Thus, both physical

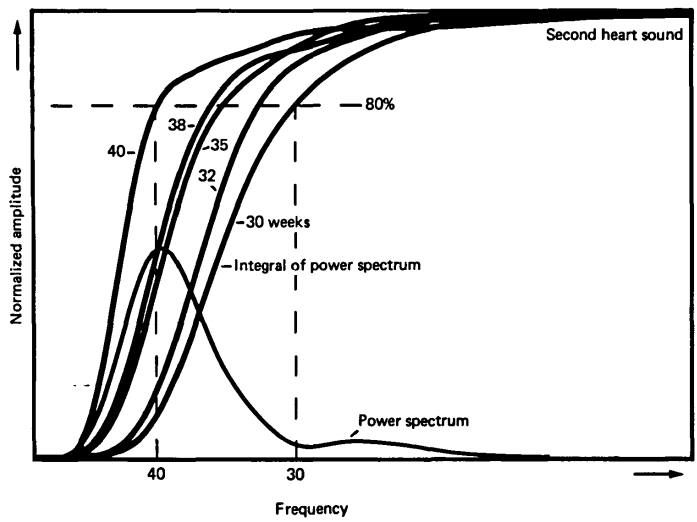

Figure 4. Normalized integrals of PCG spectra from different gestational ages 


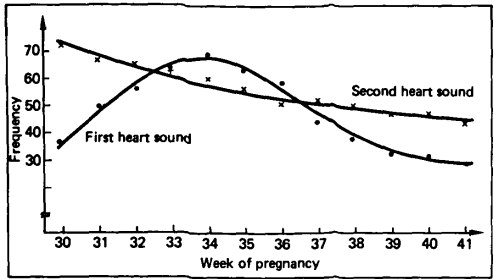

Figure 5. $80 \%$ power marks of the fetal PCG

considerations and experimental analysis show that spectral shifts can be detected best from the power spectrum integral. As a measure of spectral distribution we use that frequency, up to which $80 \%$ of the total signal power is contained. Figure 4 shows the integrated power spectra of different PCGs. The systematic shift of the $80 \%$ mark as a function of the gestational age is apparent. The mean values for the first and the second heart sound are shown in Figure 5 . There is an unequivocal relationship between the spectrum of the PCG and the week of gestation. Of course, to obtain this picture, which may serve as a calibration curve, we considered only the spectra of fetuses that were known to be developing normally. The first heart sound reveals a steady shift towards higher frequencies up to about week 34 of gestation. Thereafter the shift reverses its direction. The spectrum of the second heart sound steadily shifts to lower frequencies. The reason for the decrease in the frequencies is known to be the growth of the heart, while the ascending slope of the spectrum of the first heart sound is, according to our model, caused by a predominance of the increase in aortic valve tension that results from the increasing contractile strength of the heart muscle.

\section{Conclusions}

On the basis of these experimental results we believe that analysis of the spectrum a passive measuring technique - may be a real alternative to such well-known active measurements as ultrasonic imaging in the determination of fetal maturity. Besides the information on physical dimensions, it also contains data on the function and the strength of the heart muscle. We must, of course, also consider possible disturbing influences such as changing cardiovascular conditions (e.g. the heart rate). So far we have found no such effects. It should be mentioned that the PCGs have always been picked up at the basal heart rate with no labour activity, and not during acclerations nor decelerations. At the present time we are investigating the reliability of this method, and its applicability to routine examinations. 